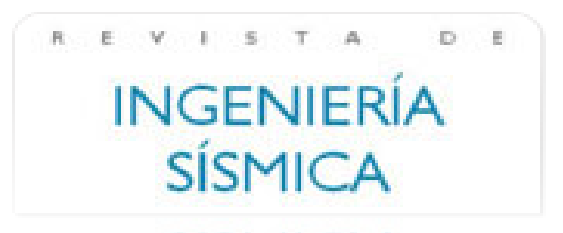

Revista de Ingeniería Sísmica

ISSN: 0185-092X

javiles@tlaloc.imta.mx

Sociedad Mexicana de Ingeniería Sísmica

México

Pineda, Omar; Ordaz, Mario

Mapas de velocidad máxima del suelo para la ciudad de México

Revista de Ingeniería Sísmica, núm. 71, julio-diciembre, 2004, pp. 37-62

Sociedad Mexicana de Ingeniería Sísmica

Distrito Federal, México

Disponible en: http://www.redalyc.org/articulo.oa?id=61807102

- Cómo citar el artículo

- Número completo

- Más información del artículo

Página de la revista en redalyc.org

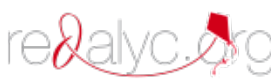

Sistema de Información Científica

Red de Revistas Científicas de América Latina, el Caribe, España y Portugal Proyecto académico sin fines de lucro, desarrollado bajo la iniciativa de acceso abierto 


\title{
MAPAS DE VELOCIDAD MÁXIMA DEL SUELO PARA LA CIUDAD DE MÉXICO
}

\author{
Omar Pineda y Mario Ordaz ${ }^{(1)}$
}

\begin{abstract}
RESUMEN
El objetivo de este artículo es presentar los mapas de distribución de velocidad máxima del suelo de la Ciudad de México para cuatro eventos sísmicos de interés. Se describe el procedimiento de cálculo, el programa de computadora empleado y su calibración, la cual permite que los valores de velocidad calculados se aproximen a los medidos. Además, se propone un grupo de ecuaciones de ajuste, en función de la magnitud del evento, con el propósito de calcular mapas de velocidades para sismos postulados. Finalmente, se presentan mapas de velocidad máxima (componentes NS y EW) para un sismo postulado con magnitud igual a 8.4 y distancia focal de $300 \mathrm{~km}$, como aplicación de las funciones de ajuste propuestas.
\end{abstract}

\section{SUMMARY}

The objective of this paper is to show peak ground velocity (PGV) maps for Mexico City during four past earthquakes. The method of computation, as well as the computer program and its calibration are described. The calibration intends to get estimated PGV values close to those measured. Additionally, a group of equations are proposed, aimed to calculate PGV maps for postulated earthquakes. Finally, PGV maps (NS and EW components) for an earthquake with magnitude equal to 8.4 and focal distance equal to $300 \mathrm{~km}$ are shown, as an application of the equations proposed.

\section{INTRODUCCIÓN}

En el proceso de diseño por sismo de estructuras convencionales se tienen en cuenta las aceleraciones espectrales máximas. Sin embargo, no todas las estructuras son sensibles a estas cantidades. En algunos casos, la velocidad máxima del suelo (VMS) está mejor correlacionada con los daños, tal como ocurre con las tuberías enterradas (Pineda y Ordaz, 2002). Algunos trabajos relacionados con el cálculo de mapas de VMS son, por ejemplo, el de Trifunac y Todorovska (1997), y el de Wu et al. (2001).

En este artículo se presenta un resumen del procedimiento seguido para determinar los mapas de distribución de VMS en la Ciudad de México para cuatro eventos sísmicos de interés.

Artículo recibido el 14 de junio de 2002 y aprobado para su publicación el 14 de abril de 2004. Se aceptarán comentarios y/o discusiones hasta cinco meses después de su publicación.

(1) Instituto de Ingeniería, UNAM, Ciudad Universitaria, 04510 México, DF. opinedap@iingen.unam.mx, mors@pumas.iingen.unam.mx 
Para este fin se utilizó una versión modificada del programa Z, desarrollado en el Instituto de Ingeniería con patrocinio del Departamento del Distrito Federal (Ordaz, 1994 y Ordaz, et al. 1996). Este programa, que inicialmente estaba encaminado a determinar espectros de respuesta, puede ahora estimar la VMS en un sitio específico de la ciudad, con las modificaciones a los modelos originales propuestas en esta publicación.

A continuación se describe brevemente la forma en que el programa Z estima el movimiento sísmico del suelo en el DF. Además, se presenta el método de cálculo de la VMS y los mapas de velocidad máxima para los sismos estudiados. Finalmente se propone una forma de ajuste del programa para estimar la VMS ante sismos postulados.

\section{DESCRIPCIÓN DEL PROGRAMA Z}

El programa Z está construido para estimar espectros de sitio en la Ciudad de México ante temblores históricos o postulados, con lo que es posible obtener espectros de respuesta ante movimientos del suelo reconocidos como temblores de diseño. A grandes rasgos, el procedimiento de estimación utilizado por el programa Z consta de los siguientes pasos: 1) Estimación del espectro de amplitudes de Fourier (EAF) en la estación acelerográfica de Ciudad Universitaria (CU), considerada como estación de referencia; 2) determinación de la función de transferencia del sitio de interés (FTS); 3) Calculo del EAF en el sitio de interés y su posterior transformación a espectro de respuesta vía la teoría de vibraciones aleatorias. En los siguientes párrafos se dan los detalles más importantes sobre este procedimiento de estimación.

\section{Cálculo del EAF en la estación de referencia}

Para estimar el EAF en la estación de referencia ante un sismo costero postulado, Ordaz et al. (1994) construyeron, a partir de registros acelerográficos obtenidos en la estación CU, un modelo de regresión bayesiana, que relaciona ordenadas del EAF a varias frecuencias con la magnitud y la distancia focal del sismo. La regresión de las amplitudes de Fourier de las aceleraciones registradas en CU preserva los rasgos prominentes debidos a la amplificación regional señalada por Ordaz y Singh, (1992), que según Singh y Ordaz (1994) está presente en todo el valle de México. Éstos EAF son estimados confiablemente en el intervalo de frecuencias entre 0.3 y $3 \mathrm{~Hz}$ debido a que sólo hay suficiente información en esa región espectral.

\section{Movimiento del terreno en las zonas de lago y transición}

Para estimar el EAF del movimiento del terreno en sitios instrumentados (en las zonas del lago y transición) Ordaz et al. (1989) propusieron el uso de funciones de transferencia empíricas de sitio (FTS). Conociendo el EAF para el sitio de referencia CU, el EAF para el sitio de interés se puede estimar mediante el producto entre la FTS y el EAF de CU. 
Las FTS fueron calculadas a partir de las amplificaciones relativas del movimiento del terreno observadas en los datos registrados por las estaciones de la Red Acelerométrica de la Ciudad de México (RACM).

Este procedimiento se ha extendido para estimar el movimiento del terreno que se tendría en sitios no instrumentados dentro del valle de México. Para estimar las FTS que se tendrían en estos sitios se desarrolló un esquema de interpolación espacial (Pérez-Rocha, 1998; Lancaster y Salkauskas, 1986; Pelto et al., 1988). En el modelo se hacen intervenir soluciones teóricas y numéricas, así como las incertidumbres relacionadas con los datos y con el tratamiento numérico, a fin de reducir y cuantificar los niveles de error que se tienen en las predicciones.

\section{MÉTODO EMPLEADO PARA EL CÁLCULO DE LA VELOCIDAD MÁXIMA DEL SUELO}

En principio, si se conoce el EAF de la aceleración del suelo en un sitio, $|A(\omega)|$, la velocidad máxima del suelo se puede estimar a partir del espectro de amplitudes de velocidad $|V(\omega)|$ cuya relación con el EAF de aceleraciones es la siguiente:

$$
|V(\omega)|=\frac{|A(\omega)|}{\omega}
$$

De forma análoga al cálculo de la aceleración máxima esperada, usando la teoría de vibraciones aleatorias (Boore y Joyner, 1984) es posible calcular la esperanza de la velocidad máxima del suelo. El valor esperado de la velocidad máxima del suelo $V$ puede escribirse como el producto de la velocidad cuadrática media $V_{c m}$ y un factor pico $F_{p}$ (ec. 2).

$$
E[V]=V_{c m} \cdot F_{p}
$$

De acuerdo con el teorema de Parseval, $V_{c m}$ puede calcularse con la siguiente ecuación:

$$
V_{c m}=\sqrt{\frac{M_{0}}{d c m}}
$$

donde $M_{o}$ es el momento de orden cero del EAV y dcm es la duración cuadrática media de la señal. Debido a que se está estimando la velocidad máxima del suelo, $d c m$ es igual a la duración de la fase intensa del temblor, la cual fue calculada con la ec. 4.

$d c m=18.9232+3.3031 \cdot M+13.6421 \cdot T+(-3.024+0.6727 \cdot M) \cdot T^{2}$

$M$ es la magnitud del evento sísmico y $T$ es el período predominante del sitio. 
Los momentos espectrales se calculan con la siguiente expresión:

$$
\underset{k=0,2 \ldots}{M_{k}}=\frac{(2 \pi)^{k-1}}{\pi} \int_{0}^{\infty} f^{k}|V(f)|^{2} d f
$$

$f$ es la frecuencia natural de vibración y es igual $\omega /(2 \pi)$. Por otra parte, $F_{p}$ puede estimarse con las ecs. 6 y 7, donde $N$ es el número estimado de cruces del registro en la línea base y $\gamma$ es la constante de Euler $(\gamma=0.5772 \ldots)$.

$$
\begin{aligned}
& F p=\sqrt{2 \cdot \ln (N)}+\frac{\gamma}{\sqrt{2 \cdot \ln (N)}} \\
& N=2 \cdot d c m \cdot \sqrt{\frac{M_{2}}{M_{0}}}
\end{aligned}
$$

Con las ecs. 1 a 7 se puede calcular la esperanza de la VMS. Sin embargo, se observó que la aplicación directa de estas ecuaciones en el programa Z conducía a subestimaciones sistemáticas de la VMS. Esto se debe a que la información contenida en el programa Z estima adecuadamente los EAF sólo en el intervalo de frecuencias de 0.3 a $3 \mathrm{~Hz}$, y la VMS es, para el valle de México, una medida de baja frecuencia.

Para la mayor parte de los acelerogramas registrados en el DF es difícil recuperar confiablemente las amplitudes espectrales para frecuencias menores de $0.3 \mathrm{~Hz}$, por lo que la reconstrucción de los EAF en terreno firme y de las FTS para baja frecuencia es sumamente difícil. Por tanto, se juzgó más conveniente realizar una calibración empírica de forma que las estimaciones de VMS sean tan parecidas como sea posible a los valores obtenidos a partir de la integración de registros sísmicos obtenidos durante varios temblores en el pasado.

Conviene hacer notar que, tal como ocurre con las otras cantidades que estima el programa Z, la estimación de VMS se llevó a cabo para las componentes NS y EW del movimiento. Se eligieron esas componentes con el propósito de no perder información al trasladar los registros sísmicos disponibles a otras direcciones, ya que la gran mayoría de ellos fueron registrados en las componentes mencionadas. Por otro lado, no se aprecian variaciones sistemáticas entre las velocidades en dos componentes que justifiquen un análisis más refinado.

\section{Eventos sísmicos estudiados}

Los eventos sísmicos usados en este trabajo para estimar la distribución de velocidades máximas fueron los ocurridos el 25 de abril de 1989, el 14 de septiembre de 1995, el 9 de octubre de 1995 y el 19 de septiembre de 1985. Los tres primeros fueron seleccionados por haber sido registrados en la mayoría de las estaciones de la RACM. El sismo de 1985 se incluyó puesto que, aunque fue registrado por pocas estaciones, ha sido el evento que ha producido las máximas velocidades del 
suelo. Se consideraron en total 152 registros sísmicos producidos por los cuatro temblores estudiados (ver tabla 1 y fig. 1).

Tabla 1. Descripción de los eventos sísmicos estudiados

\begin{tabular}{|c|c|c|c|c|}
\hline Sismo & Magnitud & $\begin{array}{c}\text { Distancia } \\
\text { focal } \\
\text { (km) }\end{array}$ & $\begin{array}{c}\text { Profundidad } \\
\text { focal } \\
\text { (km) }\end{array}$ & $\begin{array}{c}\text { Registros } \\
\text { usados }\end{array}$ \\
\hline 25/IV/89 & 6.9 & 304 & 19 & 60 \\
\hline 14/IX/95 & 7.4 & 305 & 22 & 42 \\
\hline 9/X/95 & 8.0 & 560 & 5 & 42 \\
\hline 19/IX/85 & 8.1 & 295 & 15 & 8 \\
\hline
\end{tabular}

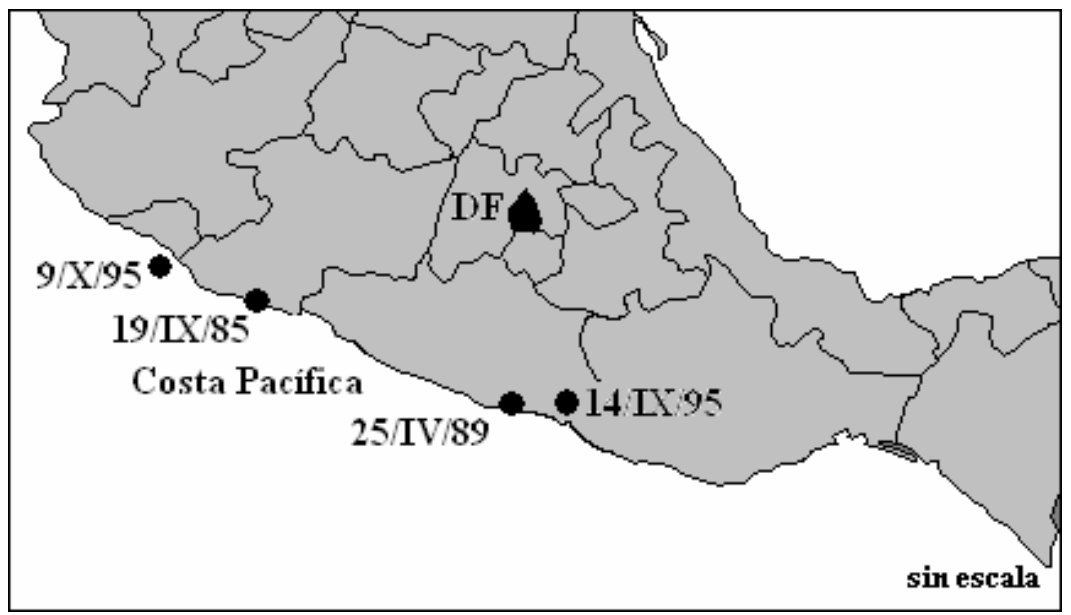

Figura 1. Localización epicentral de los sismos

De todos los registros disponibles, se realizó una selección de manera que fueron descartados aquellos de menor calidad. Por ejemplo, se desecharon aquellos en que las partes inicial o final del sismograma estuvieran alejadas de la línea base, aquellos con un número de puntos escaso, los registros segmentados en varios archivos, etc.

Aún con la selección anterior de registros, los datos tienen errores que deben ser corregidos para una mayor precisión en los cálculos. El principal error es el de la línea base, que debe coincidir con el eje horizontal. Este error se debe a desajustes en los acelerógrafos de las estaciones, principalmente inclinación no deseada del aparato. La línea base de los registros fue corregida restando una constante a todo el acelerograma, de forma que el promedio de sus valores fuera cero.

Adicionalmente se realizó un filtrado de las señales con filtros pasabanda del tipo Butterworth con 4 polos, con el propósito de tener en cuenta únicamente el intervalo de frecuencias de interés, comprendido entre 0.05 y $10 \mathrm{~Hz}$. El filtrado se realizó en el dominio de la frecuencia de acuerdo con la ec. 8 en la cual $X(\omega)$ es la transformada rápida de Fourier (TRF) 
de la señal original, $H(\omega)$ es el filtro definido por la ec. 9 (Oppenheim y Schafer, 1975) y $Y(\omega)$ es el espectro de Fourier de la señal filtrada.

$$
\begin{aligned}
& Y(\omega)=X(\omega) H(\omega) \\
& H(\omega)=\frac{1}{\sqrt{1+\left(\frac{\omega^{2}-\omega_{\text {mín }} \omega_{\text {máx }}}{\omega\left(\omega_{\text {máx }}-\omega_{\text {mín }}\right)}\right)^{2 N}}}
\end{aligned}
$$

En la ec. 9, $N$ es el número de polos, $\omega_{\min }$ y $\omega_{\operatorname{máx}}$ son las frecuencias mínima y máxima para el filtrado, que en este caso valen 0.05 y $10 \mathrm{~Hz}$ respectivamente.

Este filtrado ayuda a corregir errores de lectura de datos de los acelerógrafos asociados al ruido natural del aparato, que pueden alterar las frecuencias bajas de la señal. El límite inferior de frecuencias en el filtro pasabanda tomado fue de $0.05 \mathrm{~Hz}$ debido a que era necesario garantizar que el acelerograma llegara a cero en la velocidad final. Sin embargo, no se sabe realmente cuál es el valor del límite inferior óptimo que permita la mejor estimación de velocidad.

\section{Ajuste del programa Z}

Tal como se mencionó anteriormente, fue necesario calibrar el programa $\mathrm{Z}$ debido a la diferencia que hay entre los valores de las velocidades estimadas utilizando directamente las ecs. 1-7 y las obtenidas partiendo de los registros sísmicos. Esta diferencia se cuantifica a través del error $\varepsilon$, que definimos como el logaritmo natural del cociente entre la velocidad calculada, $V_{c}$, y la determinada a partir de la integración de los registros sísmicos vinculados en el ajuste, $V_{r}$ :

$$
\varepsilon=\ln \left(V_{c} / V_{r}\right)
$$

La forma de ajuste empleada se define con la ec. 11, donde $V_{c}$ es la velocidad calculada “corregida”, $V_{z}$ es la velocidad calculada con el programa $Z, T$ es el periodo del suelo en donde se ubica la estación acelerográfica, y finalmente, $\alpha$ y $\beta$ son constantes de ajuste.

$$
V_{c}=V_{z} \cdot e^{(\alpha \cdot T+\beta)}
$$

El objetivo del ajuste fue calcular los valores de $\alpha$ y $\beta$, para cada evento sísmico, de forma que simultáneamente la media de los valores de $\varepsilon$ valiera cero y su desviación estándar fuera mínima. La primera condición busca que se elimine el sesgo en los errores y la segunda permite controlar la dispersión de los errores en las estimaciones. 
Los períodos de suelo de los sitios donde se ubican las estaciones acelerográficas, usados para hallar las constantes de ajuste en la ec. 11, habían sido previamente determinados por otros autores (Ej: Lermo et al., 1990).

En la tabla 2 se presentan los valores de $\alpha$ y $\beta$ obtenidos al ajustar el programa Z para los sismos de interés, siguiendo el procedimiento anterior. En las tablas 3 y 4 se observa que los valores de los errores medios son prácticamente nulos y que se logró la reducción de la desviación estándar para todos los eventos y componentes; excepto en el caso de la componente EW del sismo de 1985; sin embargo, los valores de desviación estándar antes y después del ajuste son muy cercanos entre si (0.10014 y 0.10248 , respectivamente).

Tabla 2. Valores de $\alpha$ y $\beta$ para los eventos sísmicos estudiados

\begin{tabular}{|c|c|c|c|c|c|}
\hline Sismo & Magnitud & $\alpha$ NS & $\beta$ NS & $\alpha$ EW & $\beta$ EW \\
\hline $\mathbf{2 5 / I V / 8 9}$ & 6.9 & -0.1363 & 0.2855 & -0.0361 & -0.0248 \\
\hline $\mathbf{1 4 / I X / 9 5}$ & 7.4 & -0.1131 & 0.1041 & -0.1111 & 0.0839 \\
\hline $\mathbf{9 / X / 9 5}$ & 8.0 & 0.0785 & 0.2569 & 0.0978 & 0.2248 \\
\hline $\mathbf{1 9 / I X / 8 5}$ & 8.1 & -0.0126 & 0.1945 & 0.0012 & 0.1974 \\
\hline
\end{tabular}

Tabla 3. Error medio del ajuste

\begin{tabular}{|c|c|c|c|c|c|}
\hline \multirow{2}{*}{ Sismo } & \multirow{2}{*}{ No. datos } & \multicolumn{2}{|c|}{ Sin corregir } & \multicolumn{2}{c|}{ Corregidos } \\
\cline { 3 - 6 } & & NS & EW & NS & EW \\
\hline $\mathbf{2 5 / I V / 8 9}$ & 60 & -0.02823 & 0.09373 & -0.00513 & 0.00002 \\
\hline $\mathbf{1 4 / I X / 9 5}$ & 42 & 0.11569 & 0.13043 & 0.00000 & -0.00001 \\
\hline $\mathbf{9 / X / 9 5}$ & 42 & -0.43274 & -0.44174 & -0.00004 & 0.00002 \\
\hline $\mathbf{1 9 / I X / 8 5}$ & 8 & -0.17823 & -0.19895 & 0.00010 & 0.00000 \\
\hline
\end{tabular}

Tabla 4. Desviación estándar del ajuste

\begin{tabular}{|c|c|c|c|c|c|}
\hline \multirow{2}{*}{ Sismo } & \multirow{2}{*}{ No. datos } & \multicolumn{2}{|c|}{ Sin corregir } & \multicolumn{2}{c|}{ Corregidos } \\
\cline { 3 - 6 } & & NS & EW & NS & EW \\
\hline $\mathbf{2 5 / I V / 8 9}$ & 60 & 0.23328 & 0.29618 & 0.16825 & 0.27355 \\
\hline $\mathbf{1 4 / I X / 9 5}$ & 42 & 0.30587 & 0.33190 & 0.23537 & 0.25814 \\
\hline $\mathbf{9 / X / 9 5}$ & 42 & 0.19740 & 0.28401 & 0.17627 & 0.24516 \\
\hline $\mathbf{1 9 / I X / 8 5}$ & 8 & 0.24142 & 0.10014 & 0.12448 & 0.10284 \\
\hline
\end{tabular}


En la fig. 2 se muestra la distribución del error con respecto al período del suelo $T$, para cada evento sísmico. Se observa que al realizar el ajuste, la línea de tendencia de la nube de puntos es horizontal e igual a cero, lo cual indica que no hay sesgo apreciable con respecto a $T$.
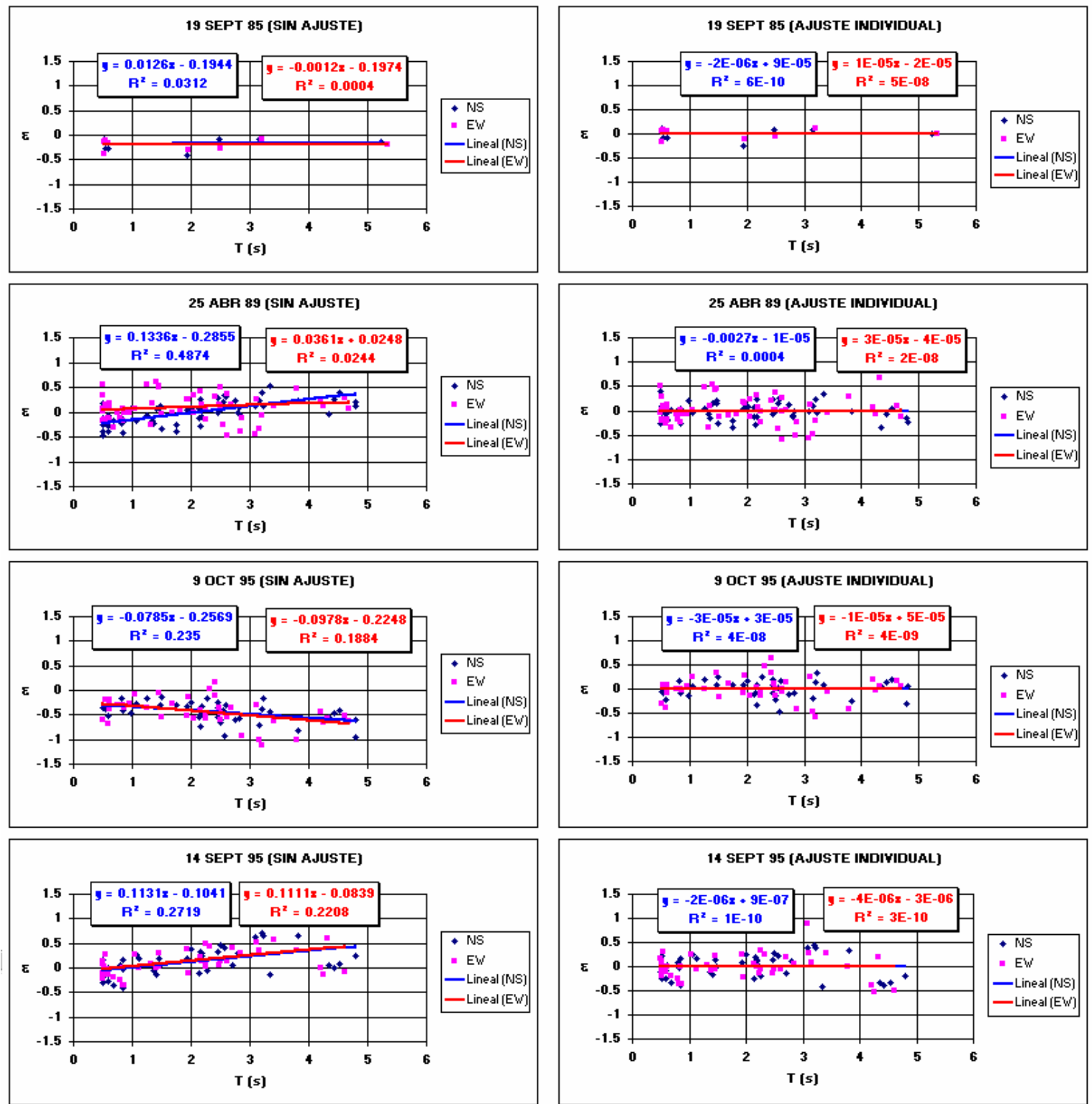

Figura 2. Distribución del error $\varepsilon$ con y sin ajuste para cada evento sísmico 


\section{MAPAS DE VELOCIDAD MÁXIMA}

Los mapas de VMS pueden ser aplicados en la estimación de daños por sismo en líneas vitales. Esto se debe a que este tipo de estructuras son afectadas por movimientos diferenciales del suelo, que se ha demostrado son proporcionales a la velocidad del suelo (Newmark, 1967). Al estimar los valores de VMS se está cuantificando el valor máximo esperado de este parámetro sísmico, y de esta forma, se puede estimar el daño máximo esperado en este tipo de estructuras. Un ejemplo de la aplicación de este tipo de mapas es el presentado por Pineda y Ordaz (2003), quienes estimaron empíricamente el posible daño en la red primaria de distribución de agua potable del Distrito Federal debido a sismos futuros.

Las figs. 3 a 10 corresponden a los mapas de VMS para los eventos sísmicos estudiados, los cuales fueron obtenidos con el programa $\mathrm{Z}$ y los valores de las constantes $\alpha$ y $\beta$ (tabla 2), de acuerdo con la ec. 11. En la tabla 5 se presentan tanto los valores de velocidad obtenidos a partir de la integración de los registros sísmicos, para cada evento sísmico, con los calculados con el procedimiento descrito.

En términos generales, en los mapas se observa que los valores de VMS son superiores en la zona de lago que en el resto de la ciudad debido a la amplificación dinámica característica de los depósitos lacustres presentes en esa región.

Al comparar los mapas de los sismos ocurridos el 25/IV/89, 14/IX/95 y 19/IX/85 (caracterizados por tener distancia focal similar) se observa que los valores de VMS aumentan a medida que aumenta la magnitud. Con respecto al sismo del 9/X/95 se puede decir que sus valores de VMS son menores a los presentados por los otros mapas debido a que su distancia focal es superior (560 km) en comparación con la de los otros sismos (alrededor de $300 \mathrm{~km}$ ).

\section{AJUSTE DEL PROGRAMA Z PARA SISMOS POSTULADOS}

Como se ha dicho antes, la aplicación directa de las ecs. 1 a 7 en el programa Z no conduce a resultados suficientemente precisos, por lo que es necesario aplicar correcciones empíricas. Hasta ahora, se ha presentado la obtención de estas correcciones empíricas para cuatro sismos, individualmente. Sin embargo, para conocer la distribución de velocidades máximas del suelo para eventos sísmicos postulados, es necesario relacionar las constantes de ajuste ( $\alpha$ y $\beta$ ) con los parámetros que caracterizan un sismo, por ejemplo: magnitud, distancia y profundidad focal, entre otros.

Para este fin, en este artículo se determinaron valores de $\alpha$ y $\beta$ para sismos postulados, como función de un solo parámetro: la magnitud $m$. Se hizo depender los factores correctivos sólo de $m$ ya que la información disponible proviene de sólo cuatro eventos sísmicos, por lo que no sería apropiado calcular funciones de ajuste considerando más de un parámetro sin suficientes datos. Se descartó también la posibilidad de incluir la distancia focal en el análisis ya que los valores de esta cantidad para tres de los sismos están alrededor de los $300 \mathrm{~km}$. 


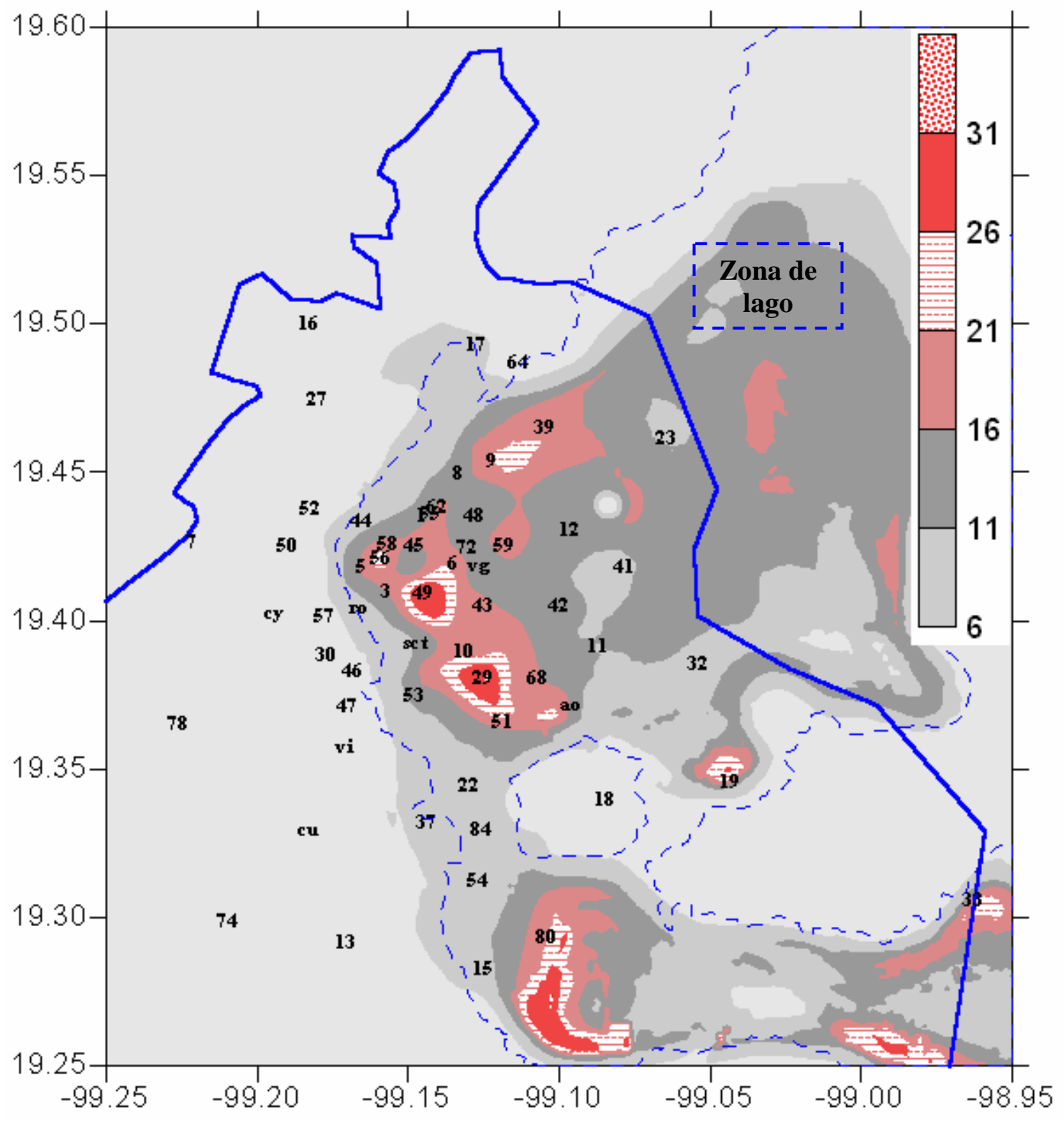

Figura 3. Mapa de VMS para el sismo del 25 de abril de 1989 (N-S) (cm/s) 


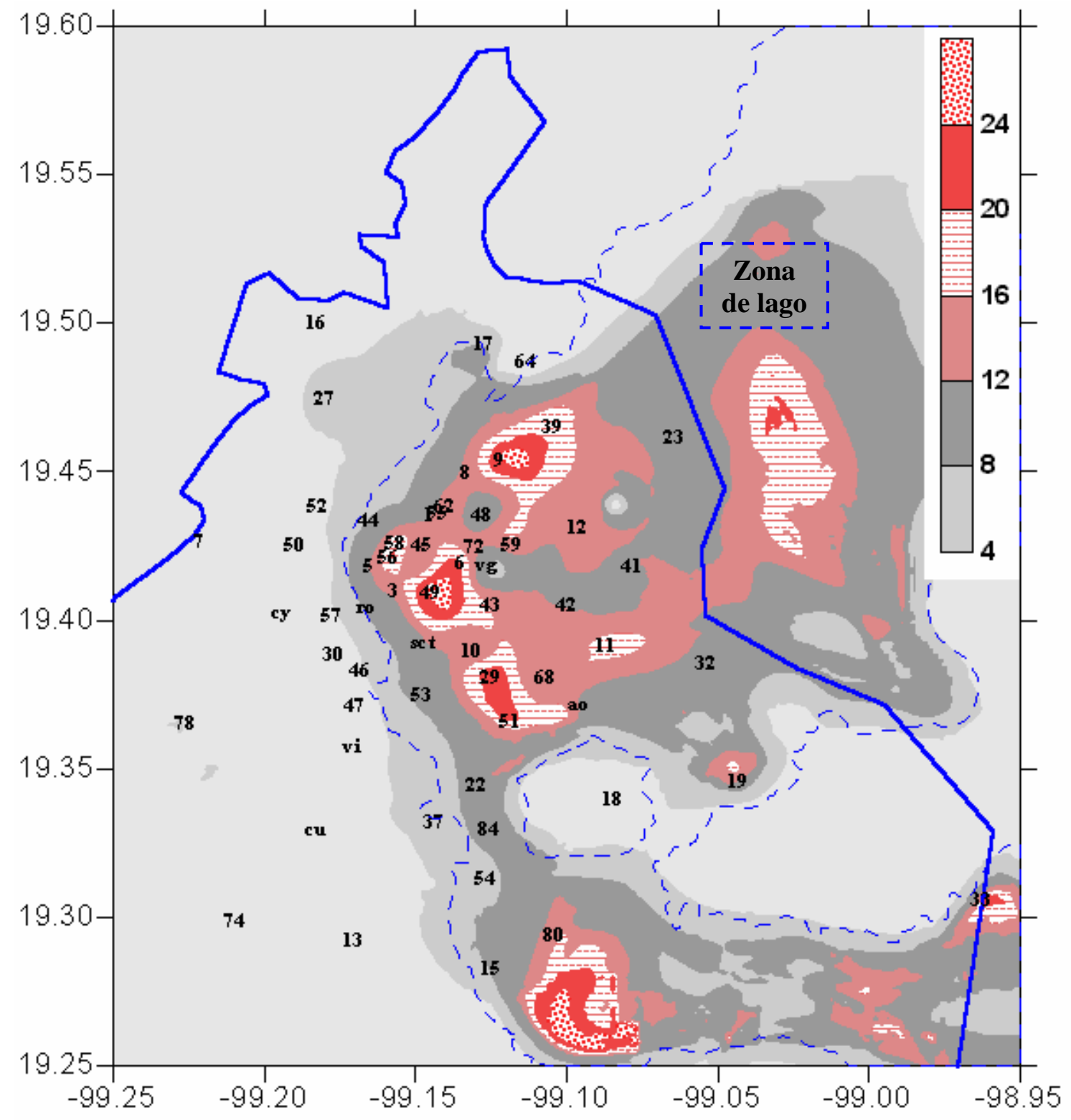

Figura 4. Mapa de VMS para el sismo del 25 de abril de 1989 (E-W) (cm/s) 


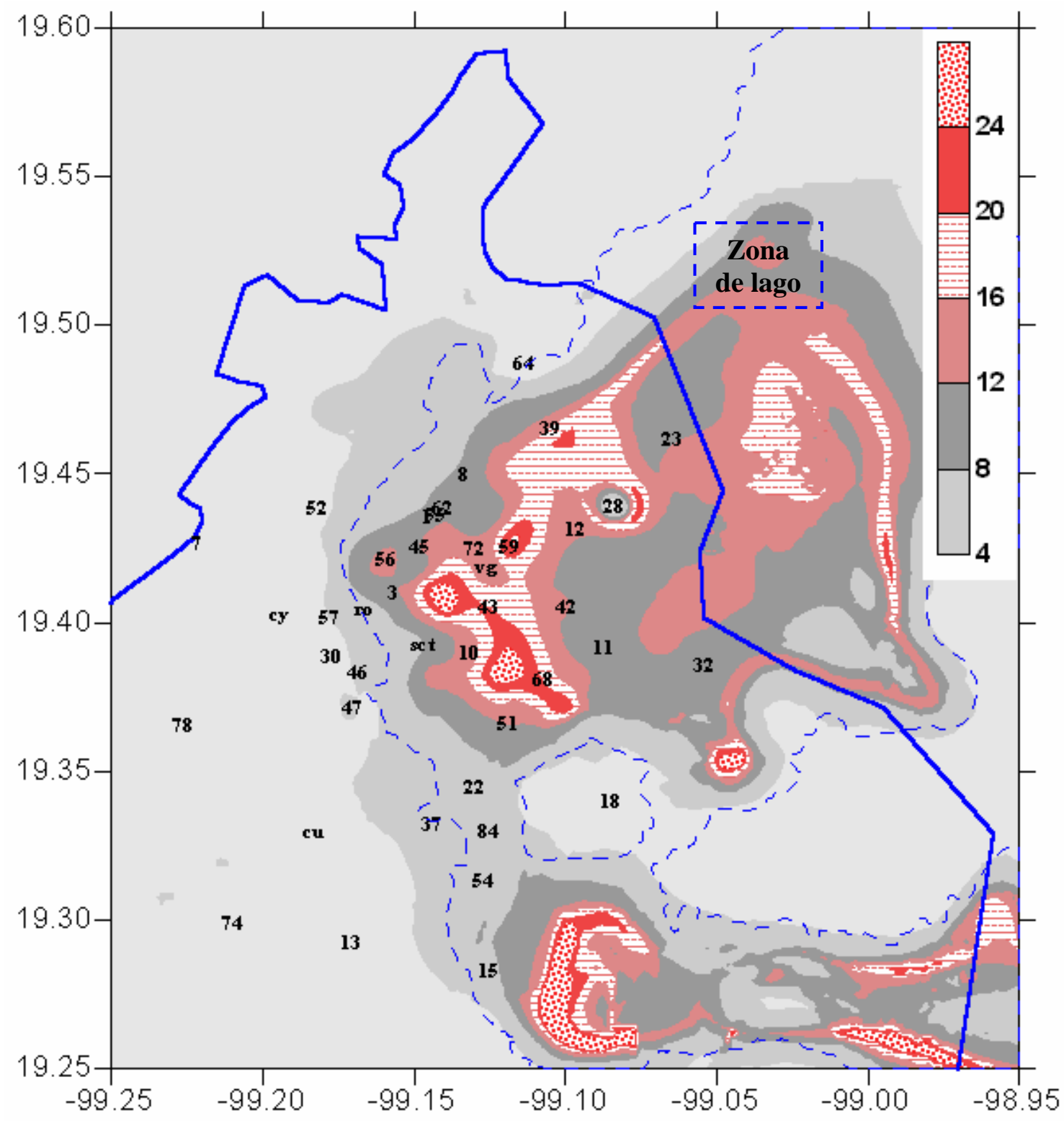

Figura 5. Mapa de VMS para el sismo del 14 de septiembre de 1995 (N-S) (cm/s) 


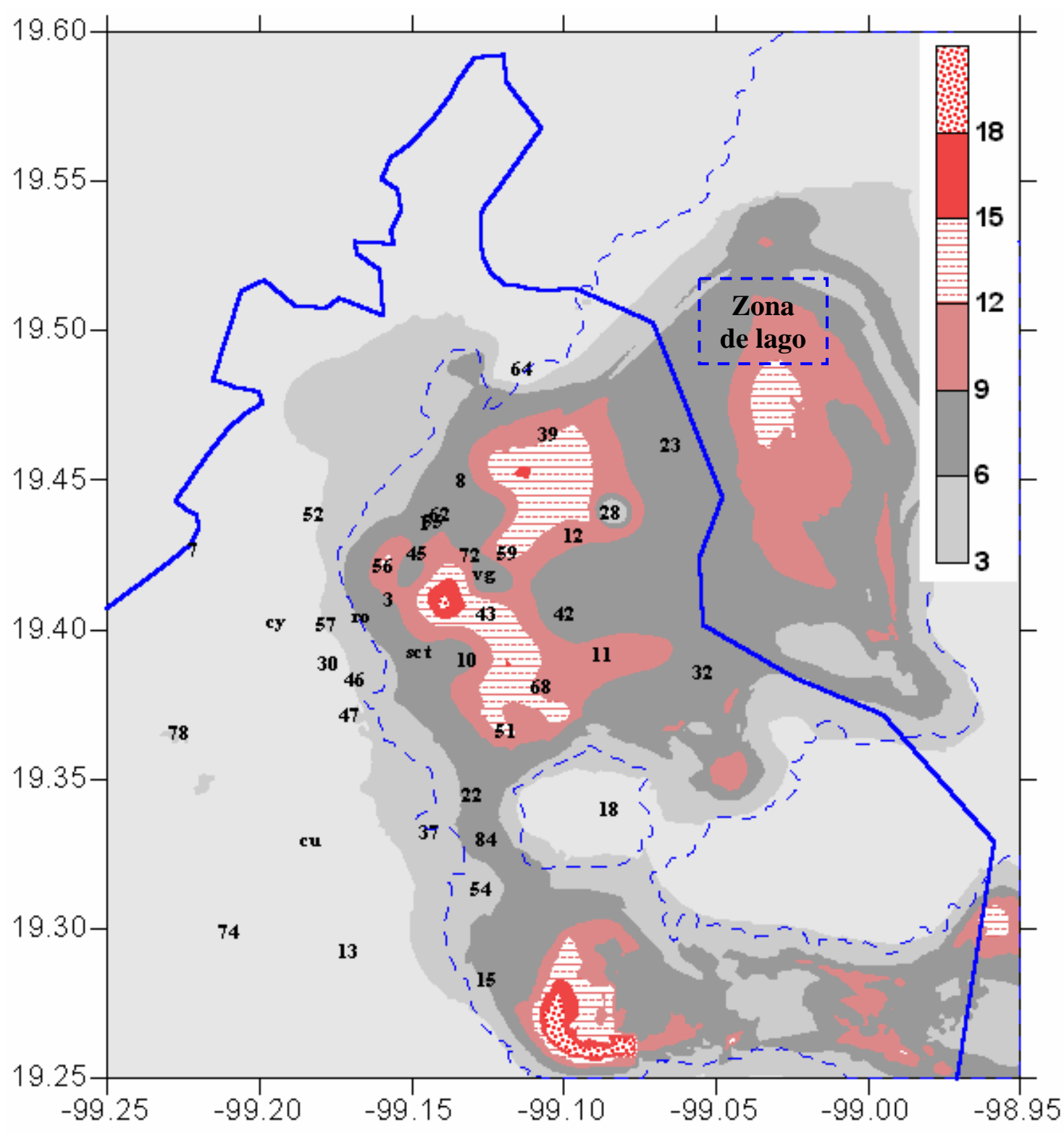

Figura 6. Mapa de VMS para el sismo del 14 de septiembre de 1995 (E-W) (cm/s) 


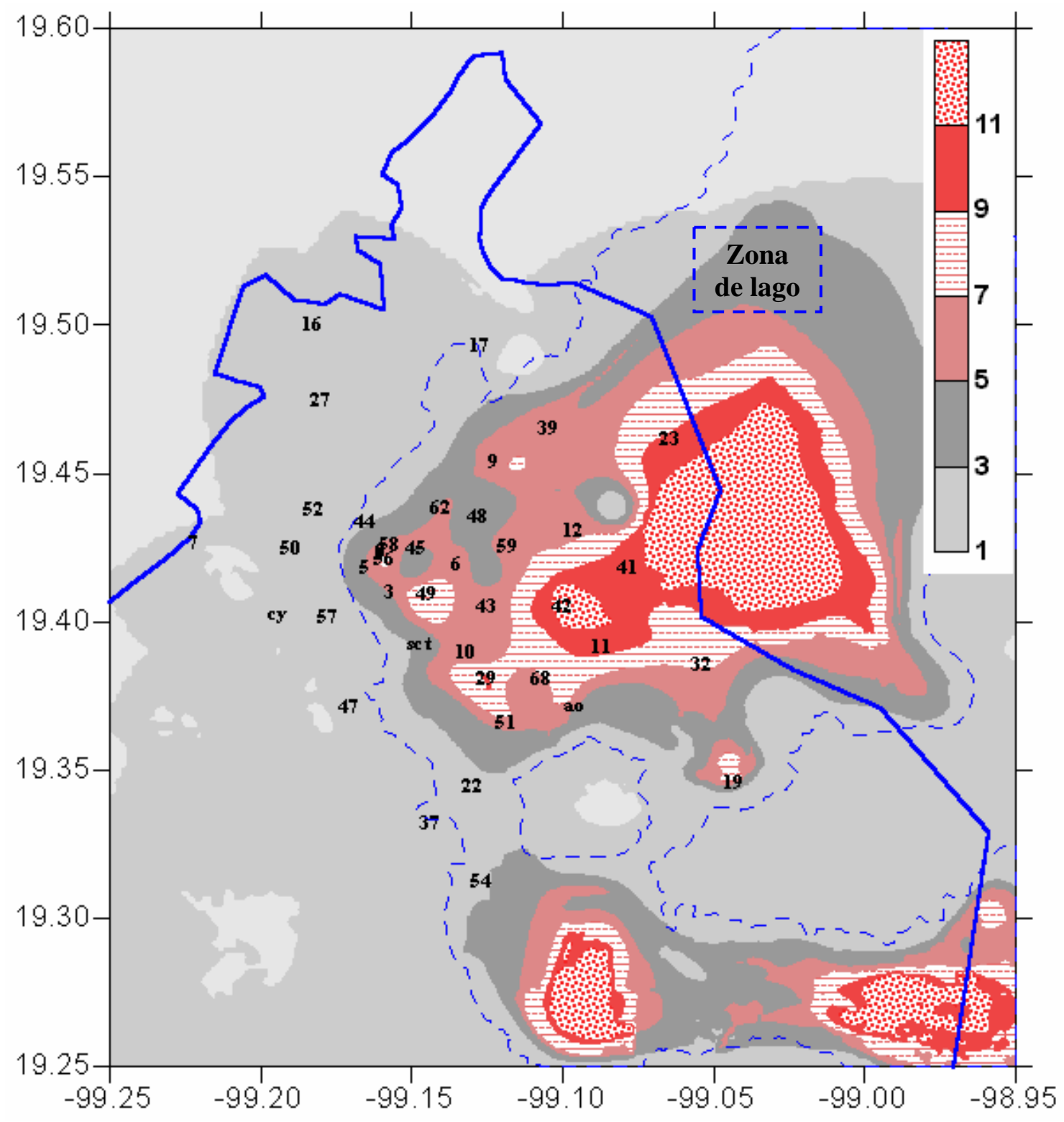

Figura 7. Mapa de VMS para el sismo del 9 de octubre de 1995 (N-S) (cm/s) 


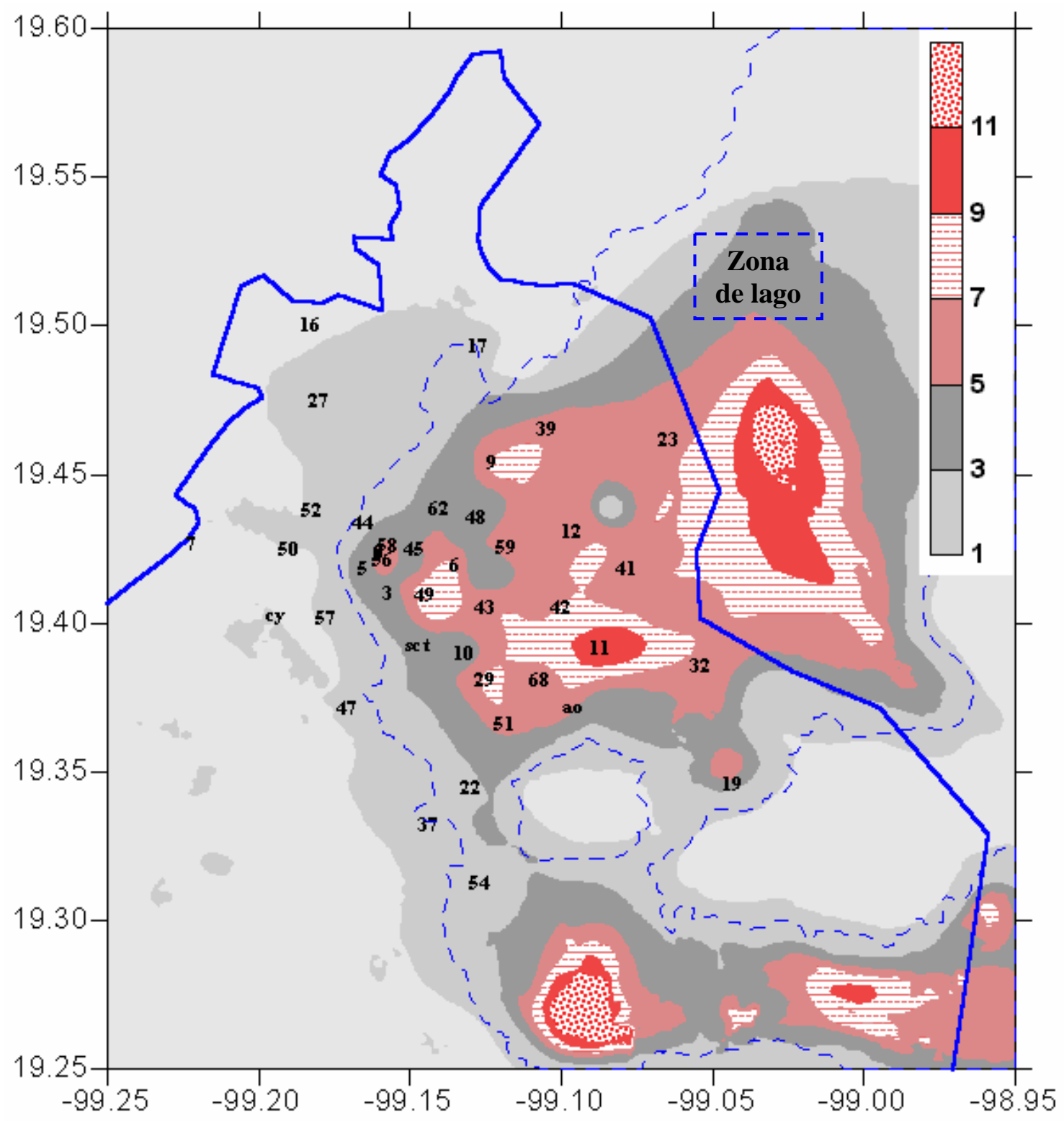

Figura 8. Mapa de VMS para el sismo del 9 de octubre de 1995 (E-W) (cm/s) 


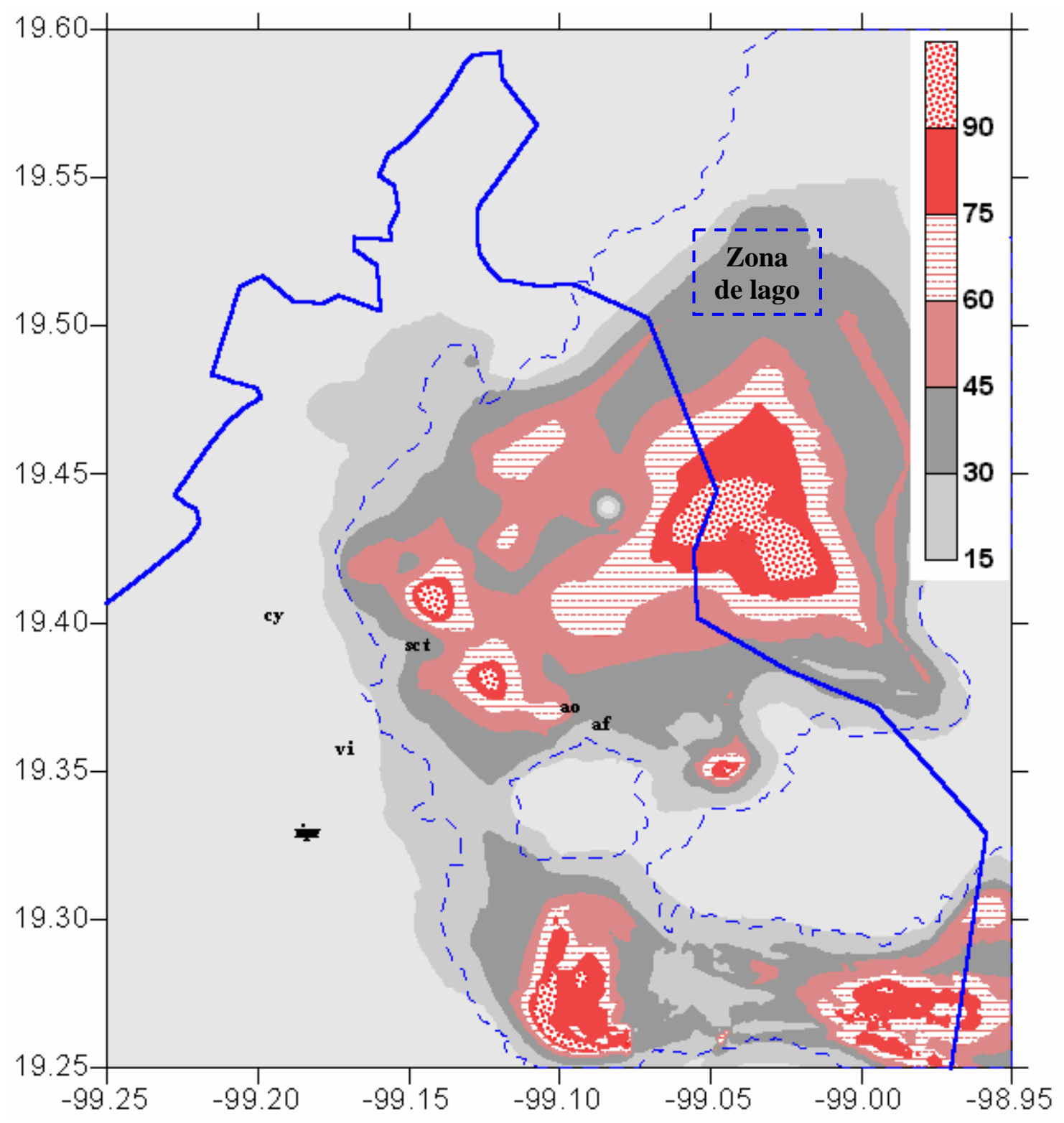

Figura 9. Mapa de VMS para el sismo del 19 de septiembre de 1985 (N-S) (cm/s) 


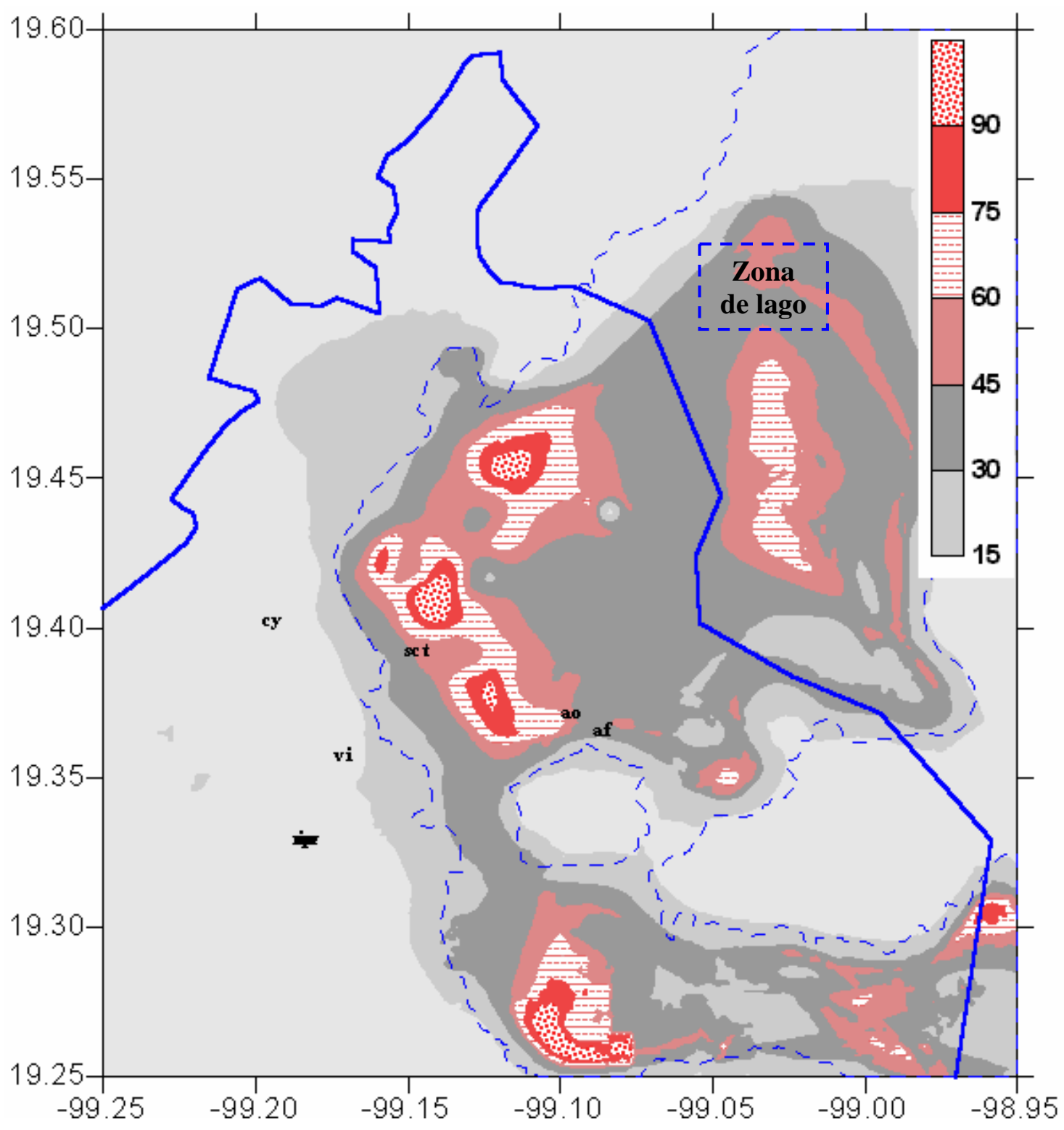

Figura 10. Mapa de VMS para el sismo del 19 de septiembre de 1985 (E-W) (cm/s) 
Omar Pineda y Mario Ordaz

Tabla 5. Valores de velocidad máxima del suelo (cm/s) calculados con el programa Z (Vc) y obtenidos por integración (Vr)

\begin{tabular}{|c|c|c|c|c|c|c|c|c|c|c|c|c|c|c|c|c|}
\hline Sismos & \multicolumn{4}{|c|}{ 25/IV/89 } & \multicolumn{4}{|c|}{ 14/IX/95 } & \multicolumn{4}{|c|}{ 9/X/95 } & \multicolumn{4}{|c|}{ 19/IX/85 } \\
\hline \multirow{2}{*}{ Estación } & \multicolumn{2}{|c|}{ NS } & \multicolumn{2}{|c|}{ EW } & \multicolumn{2}{|c|}{ NS } & \multicolumn{2}{|c|}{ EW } & \multicolumn{2}{|c|}{ NS } & \multicolumn{2}{|c|}{ EW } & \multicolumn{2}{|c|}{ NS } & \multicolumn{2}{|c|}{ EW } \\
\hline & $V c$ & $V r$ & $V c$ & $V r$ & $V c$ & $V r$ & $V c$ & $V r$ & $V c$ & $V r$ & $V c$ & $V r$ & $V c$ & $V r$ & $V c$ & $V r$ \\
\hline Al01 & 13.34 & 15.00 & 11.00 & 10.40 & 8.72 & 11.30 & 7.03 & 7.13 & - & - & - & - & - & - & - & - \\
\hline Ap68 & 5.08 & 10.70 & 11.65 & 12.00 & 17.17 & 26.10 & 9.84 & 7.54 & 6.96 & 6.40 & 6.01 & 6.41 & - & - & - & - \\
\hline Au11 & 9.54 & 9.08 & 14.61 & 7.45 & 8.71 & 12.10 & 8.48 & 6.90 & 9.83 & 8.05 & 10.12 & 9.51 & - & - & - & - \\
\hline Au46 & 5.57 & 5.47 & 3.84 & 4.53 & 4.42 & 4.09 & 3.22 & 4.10 & - & - & - & - & - & - & - & - \\
\hline Ba49 & 27.29 & 23.90 & 22.84 & 22.50 & - & - & - & - & 8.29 & 9.31 & 7.82 & 6.80 & - & - & - & - \\
\hline Bl45 & 15.22 & 16.10 & 14.09 & 13.90 & 11.01 & 9.47 & 8.14 & 8.60 & 4.96 & 4.05 & 4.78 & 4.79 & - & - & - & - \\
\hline Bo39 & 19.21 & 14.60 & 17.31 & 17.70 & 15.19 & 12.10 & 10.24 & 10.90 & 5.92 & 9.50 & 6.02 & 6.06 & - & - & - & - \\
\hline Ca59 & 17.35 & 13.80 & 13.76 & 22.30 & 21.23 & 13.60 & 11.65 & 10.70 & 6.44 & 5.64 & 6.08 & 9.88 & - & - & - & - \\
\hline Cdaf & - & - & - & - & - & - & - & - & - & - & - & - & 25.79 & 23.90 & 33.72 & 36.00 \\
\hline Cdao & 13.92 & 12.80 & 10.95 & 13.40 & - & - & - & - & 5.96 & 7.30 & 5.34 & 9.57 & 36.19 & 33.50 & 44.64 & 40.10 \\
\hline Ce18 & 2.45 & 2.43 & 1.18 & 1.68 & 2.07 & 2.86 & 1.12 & 1.36 & - & - & - & - & - & - & - & - \\
\hline Ce23 & 9.00 & 10.50 & 8.83 & 8.04 & 9.10 & 11.00 & 5.69 & 9.50 & 9.45 & 12.90 & 6.12 & 5.20 & - & - & - & - \\
\hline Ce32 & 7.94 & 11.20 & 9.04 & 9.58 & 7.33 & 10.20 & 5.69 & 9.52 & 7.31 & 6.87 & 6.11 & 6.26 & - & - & - & - \\
\hline Ch84 & 8.67 & 7.41 & 10.94 & 6.45 & 5.79 & 6.93 & 7.04 & 7.57 & - & - & - & - & - & - & - & - \\
\hline Ci05 & 16.27 & 20.20 & 11.84 & 12.90 & - & - & - & - & 4.98 & 4.55 & 3.93 & 3.48 & - & - & - & - \\
\hline Cj03 & 15.50 & 13.90 & 12.65 & 10.70 & 10.09 & 7.82 & 8.29 & 6.44 & 4.99 & 4.21 & 4.36 & 4.78 & - & - & - & - \\
\hline Co47 & 4.90 & 5.18 & 3.62 & 3.22 & 4.19 & 4.02 & 3.06 & 3.09 & 1.52 & 1.53 & 1.23 & 1.34 & - & - & - & - \\
\hline Co56 & 20.56 & 21.00 & 18.72 & 12.90 & 13.25 & 12.50 & 11.39 & 10.80 & 7.33 & 8.46 & 5.93 & 6.73 & - & - & - & - \\
\hline Cp28 & - & - & - & - & 2.36 & 2.71 & 2.25 & 1.87 & - & - & - & - & - & - & - & - \\
\hline Cs78 & 3.75 & 3.89 & 2.25 & 1.68 & 3.21 & 4.09 & 2.04 & 2.24 & - & - & - & - & - & - & - & - \\
\hline Ct64 & 1.30 & 1.68 & 1.02 & 1.29 & 1.08 & 0.91 & 0.92 & 0.93 & - & - & - & - & - & - & - & - \\
\hline Cu01 & 4.31 & 4.41 & 2.52 & 3.03 & 3.31 & 3.76 & 2.21 & 2.12 & - & - & - & - & 10.26 & 9.32 & 9.59 & 8.98 \\
\hline Cu80 & 19.41 & 22.00 & 13.79 & 19.80 & - & - & - & - & - & - & - & - & - & - & - & - \\
\hline Cuip & - & - & - & - & - & - & - & - & - & - & - & - & 10.26 & 9.28 & 9.59 & 9.08 \\
\hline Cumv & - & - & - & - & - & - & - & - & - & - & - & - & 10.26 & 9.60 & 9.59 & 11.40 \\
\hline Dfro & .11 & 10.50 & 9.09 & 12.70 & 6.60 & 7.33 & 6.56 & 6.45 & - & - & - & - & - & - & - & - \\
\hline Dfvg & 11.61 & 11.80 & 7.79 & 13.80 & 12.28 & 8.43 & 6.31 & 2.56 & - & - & - & - & - & - & - & - \\
\hline Dm12 & 11.30 & 11.40 & 11.83 & 8.77 & 11.74 & 8.53 & 8.64 & 8.53 & 6.39 & 8.21 & 6.08 & 9.19 & - & - & - & - \\
\hline Dr16 & 3.97 & 3.68 & 3.21 & 3.93 & - & - & - & - & 1.14 & 1.13 & 0.94 & 0.87 & - & - & - & - \\
\hline Dx37 & 7.33 & 7.68 & 6.15 & 6.07 & 5.74 & 4.43 & 4.56 & 3.60 & 2.35 & 2.21 & 1.82 & 1.41 & - & - & - & - \\
\hline Eo30 & 3.93 & 4.26 & 2.90 & 3.75 & 3.25 & 3.57 & 2.38 & 2.65 & - & - & - & - & - & - & - & - \\
\hline Es57 & 5.06 & 4.89 & 3.81 & 3.72 & 4.11 & 4.18 & 3.14 & 4.51 & 1.53 & 1.53 & 1.20 & 1.19 & - & - & - & - \\
\hline Ex08 & - & - & - & - & - & - & - & - & 4.34 & 5.98 & 3.93 & 4.13 & - & - & - & - \\
\hline
\end{tabular}


Mapas de Velocidad Máxima del Suelo para la Ciudad de México

\begin{tabular}{|c|c|c|c|c|c|c|c|c|c|c|c|c|c|c|c|c|}
\hline \multicolumn{17}{|c|}{ Tabla 5. (Continuación) } \\
\hline \multirow{3}{*}{$\begin{array}{c}\text { Sismos } \\
\text { Estación }\end{array}$} & \multicolumn{4}{|c|}{ 25/IV/89 } & \multicolumn{4}{|c|}{ 14/IX/95 } & \multicolumn{4}{|c|}{ 9/X/95 } & \multicolumn{4}{|c|}{ 19/IX/85 } \\
\hline & \multicolumn{2}{|c|}{ NS } & \multicolumn{2}{|c|}{$\mathbf{E W}$} & \multicolumn{2}{|c|}{ NS } & \multicolumn{2}{|c|}{$\mathbf{E W}$} & \multicolumn{2}{|c|}{ NS } & \multicolumn{2}{|c|}{ EW } & \multicolumn{2}{|c|}{ NS } & \multicolumn{2}{|c|}{ EW } \\
\hline & $V c$ & $V r$ & Vc & $V r$ & $V c$ & $\boldsymbol{V r}$ & Vc & $V r$ & Vc & $V r$ & Vc & $V r$ & Vc & $V r$ & $V c$ & $V r$ \\
\hline Ex09 & - & - & - & - & - & - & - & - & 6.26 & 5.69 & 6.96 & 3.69 & - & - & - & - \\
\hline Fj74 & 3.10 & 3.72 & 2.37 & 2.92 & 2.47 & 2.68 & 1.99 & 2.40 & - & - & - & - & - & - & - & - \\
\hline Ga62 & 15.52 & 17.40 & 12.77 & 12.90 & 9.97 & 8.90 & 7.74 & 8.29 & 5.19 & 4.73 & 4.17 & 4.73 & - & - & - & - \\
\hline Gr27 & 5.46 & 5.82 & 4.84 & 5.16 & - & - & - & - & 1.57 & 1.33 & 1.36 & 1.35 & - & - & - & - \\
\hline Ha41 & 9.13 & 11.60 & 9.97 & 11.40 & - & - & - & - & 10.14 & 9.68 & 6.04 & 5.72 & - & - & - & - \\
\hline Hj72 & 13.46 & 14.80 & 12.24 & 20.40 & 11.82 & 10.70 & 7.93 & 7.49 & - & - & - & - & - & - & - & - \\
\hline Ib22 & 8.66 & 7.05 & 9.99 & 6.36 & 6.13 & 6.11 & 6.42 & 6.75 & 2.73 & 2.52 & 2.85 & 2.06 & - & - & - & - \\
\hline Ja43 & 15.33 & 16.00 & 14.21 & 12.60 & 17.71 & 11.90 & 12.86 & 9.84 & 5.86 & 4.19 & 6.43 & 5.06 & - & - & - & - \\
\hline Jc54 & 8.96 & 8.60 & 7.86 & 4.80 & 6.67 & 5.70 & 5.42 & 5.69 & 3.08 & 2.52 & 2.46 & 2.47 & - & - & - & - \\
\hline Li33 & 16.82 & 15.60 & 14.40 & 16.30 & - & - & - & - & - & - & - & - & - & - & - & - \\
\hline Li58 & 16.57 & 22.20 & 16.96 & 12.30 & - & - & - & - & 5.53 & 5.93 & 5.23 & 5.04 & - & - & - & - \\
\hline Lv17 & 8.87 & 7.12 & 7.94 & 8.46 & - & - & - & - & 2.47 & 2.85 & 2.39 & 2.26 & - & - & - & - \\
\hline Me52 & 4.38 & 4.59 & 3.55 & 3.79 & 3.43 & 5.09 & 2.81 & 4.07 & 1.32 & 1.42 & 1.09 & 1.13 & - & - & - & - \\
\hline Mi15 & 10.76 & 9.35 & 9.23 & 6.07 & 7.82 & 6.82 & 6.58 & 5.26 & - & - & - & - & - & - & - & - \\
\hline Mt50 & 4.21 & 5.06 & 3.05 & 3.03 & - & - & - & - & 1.25 & 1.23 & 1.05 & 1.07 & - & - & - & - \\
\hline My19 & 18.31 & 17.10 & 12.86 & 10.10 & - & - & - & - & 6.01 & 6.76 & 4.22 & 4.88 & - & - & - & - \\
\hline Pa34 & 3.80 & 3.34 & 2.74 & 2.61 & 2.88 & 3.68 & 2.32 & 2.52 & 1.17 & 1.47 & 0.89 & 1.31 & - & - & - & - \\
\hline Pd42 & 12.07 & 13.00 & 10.39 & 9.72 & 10.09 & 14.80 & 6.29 & 9.22 & 12.81 & 11.20 & 6.67 & 5.45 & - & - & - & - \\
\hline Pe10 & 18.95 & 18.60 & 12.84 & 12.50 & 13.23 & 10.00 & 7.79 & 8.92 & 5.82 & 7.21 & 4.29 & 3.86 & - & - & - & - \\
\hline Rm48 & 11.75 & 10.10 & 10.30 & 18.80 & - & - & - & - & 4.00 & 3.84 & 3.78 & 4.05 & - & - & - & - \\
\hline Sct1 & 11.44 & 10.90 & 11.46 & 9.80 & 7.58 & 7.06 & 7.50 & 9.49 & 3.69 & 3.40 & 3.89 & 2.98 & 29.16 & 37.10 & 52.38 & 58.70 \\
\hline Si53 & 8.60 & 9.22 & 8.57 & 7.72 & - & - & - & - & - & - & - & - & - & - & - & - \\
\hline Sp51 & 24.73 & 16.80 & 21.46 & 12.80 & 11.42 & 13.90 & 11.68 & 9.10 & 6.61 & 5.08 & 6.22 & 3.88 & - & - & - & - \\
\hline Sxvi & 4.34 & 5.28 & 2.84 & 3.38 & - & - & - & - & - & - & - & - & 10.22 & 11.20 & 11.51 & 11.00 \\
\hline Тасу & 3.77 & 3.58 & 2.68 & 2.40 & 3.15 & 3.22 & 2.39 & 1.78 & 1.28 & 1.38 & 0.98 & 0.90 & 9.76 & 10.60 & 10.06 & 9.30 \\
\hline Te07 & 3.40 & 3.11 & 2.49 & 2.46 & 2.91 & 2.34 & 2.20 & 1.96 & 1.07 & 1.14 & 0.88 & 1.21 & - & - & - & - \\
\hline Tl08 & 13.38 & 10.50 & 12.33 & 15.70 & 9.04 & 7.50 & 7.39 & 8.58 & - & - & - & - & - & - & - & - \\
\hline Tl55 & 8.75 & 12.30 & 8.91 & 9.53 & 9.54 & 8.22 & 7.32 & 5.26 & - & - & - & - & - & - & - & - \\
\hline Tp13 & 4.24 & 5.44 & 2.77 & 2.86 & 3.41 & 3.81 & 2.43 & 2.07 & - & - & - & - & - & - & - & - \\
\hline Uc44 & 8.75 & 11.40 & 8.05 & 9.07 & - & - & - & - & 2.78 & 2.17 & 2.44 & 2.87 & - & - & - & - \\
\hline Vg09 & 19.41 & 15.50 & 22.17 & 18.20 & - & - & - & - & 6.26 & 6.82 & 6.96 & 4.93 & - & - & - & - \\
\hline Vm29 & 29.22 & 22.70 & 20.69 & 16.00 & - & - & - & - & 9.02 & 7.60 & 7.01 & 7.22 & - & - & - & - \\
\hline Xp06 & 18.48 & 18.80 & 18.39 & 23.50 & - & - & - & - & 6.07 & 6.62 & 6.72 & 10.20 & - & - & - & - \\
\hline
\end{tabular}


En la tabla 6 y fig. 11 se presentan las ecuaciones de ajuste para sismos postulados, las cuales vinculan en el cálculo dos parámetros que describen al sismo y al sitio: la magnitud y el período predominante del suelo. Estas ecuaciones fueron calculadas al buscar que el error medio ponderado $\varepsilon_{m p}$ (ec. 12) fuera igual a cero y la desviación estándar ponderada $\sigma_{p}$ (ec. 13) fuera la mínima posible.

Tabla 6. Ecuaciones de ajuste para sismos postulados

\begin{tabular}{|c|c|c|}
\hline & $m \leq 7.4$ & $m>7.4$ \\
\hline$\alpha$ & -0.1 & $0.142857 \cdot m-1.157142$ \\
\hline$\beta$ & 0.1 & $0.142857 \cdot m-0.957142$ \\
\hline
\end{tabular}

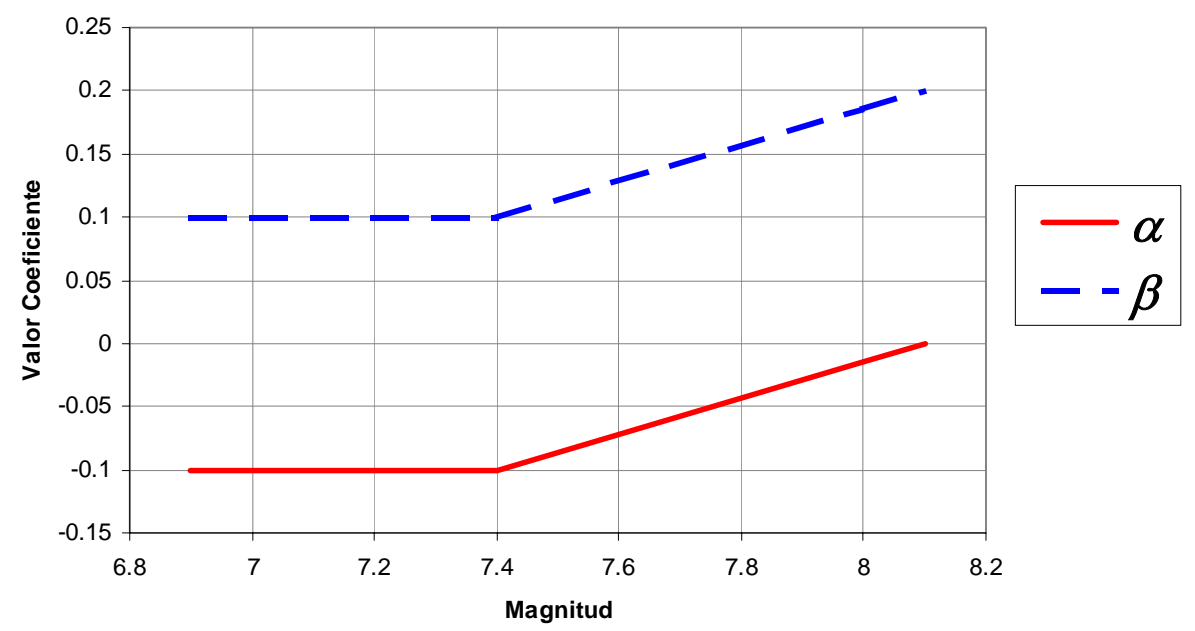

Figura 11. Variación de los coeficientes $\alpha$ y $\beta$ para sismos postulados

$$
\begin{gathered}
\varepsilon_{m p}=\frac{\sum_{i=1}^{N E} \varepsilon_{i} \cdot N_{i}}{\sum_{i=1}^{N E} N_{i}} \\
\sigma_{p}=\frac{\sum_{i=1}^{N E} \sigma_{i} \cdot N_{i}}{\sum_{i=1}^{N E} N_{i}}
\end{gathered}
$$

En las ecs. 12 y 13, $\varepsilon_{i}$ y $\sigma_{i}$ son los valores del error medio y desviación estándar de cada evento sísmico, respectivamente, $N_{i}$ es el número de datos del evento $i$ y $N E$ es el número de eventos considerados en el ajuste, que en este caso es cuatro. 
En las tablas 7 y 8 se observa el valor del error medio ponderado y la desviación estándar del ajuste de los datos. Por una parte, se logró que el error medio ponderado $\varepsilon_{m p}$ fuera cercano a cero luego del ajuste general y que adicionalmente se redujera el valor absoluto del error medio individual de cada evento. Por otra parte, se logró disminuir tanto la desviación estándar ponderada $\sigma_{p}$ como el valor individual de cada sismo, con dos excepciones. El valor de desviación estándar que no se logró reducir corresponde a la componente NS del sismo del 9 de octubre de 1995, sin embargo su diferencia es sólo 0.01333. Esto puede deberse a que la distancia focal de este sismo es mayor que la de los otros eventos. El otro valor que no se logró reducir fue el de la componente EW para el sismo del 19 de septiembre de 1985; sin embargo, al comparar los errores antes y después del ajuste se observa que, en términos prácticos, son iguales.

Tabla 7. Error medio del ajuste general

\begin{tabular}{|c|c|c|c|c|c|}
\hline \multirow{2}{*}{ Sismo } & \multirow{2}{*}{ No. datos } & \multicolumn{2}{|c|}{ Sin corregir } & \multicolumn{2}{|c|}{ Con ajuste general } \\
\hline & & NS & EW & NS & EW \\
\hline 25/IV/89 & 60 & -0.02823 & 0.09373 & -0.02821 & 0.02473 \\
\hline 14/IX/95 & 42 & 0.11569 & 0.13043 & 0.02136 & 0.03750 \\
\hline 9/X/95 & 42 & -0.43274 & -0.44174 & -0.27902 & -0.28771 \\
\hline 19/IX/85 & 8 & -0.17823 & -0.19895 & 0.02177 & 0.00105 \\
\hline \multicolumn{2}{|c|}{$\begin{array}{c}\text { Error medio } \\
\text { ponderado }\end{array}$} & -0.10813 & -0.05949 & -0.08119 & -0.05932 \\
\hline
\end{tabular}

Tabla 8. Desviación estándar del ajuste general

\begin{tabular}{|c|c|c|c|c|c|}
\hline \multirow{2}{*}{ Sismo } & \multirow{2}{*}{ No. datos } & \multicolumn{2}{|c|}{ Sin corregir } & \multicolumn{2}{c|}{ Con ajuste general } \\
\cline { 3 - 6 } & & NS & EW & NS & EW \\
\hline 25/IV/89 & 60 & 0.23328 & 0.29618 & 0.17321 & 0.28402 \\
\hline $\mathbf{1 4 / I X / 9 5}$ & 42 & 0.30587 & 0.33190 & 0.23595 & 0.25851 \\
\hline $\mathbf{9 / X / 9 5}$ & 42 & 0.19740 & 0.28401 & 0.21073 & 0.28006 \\
\hline $\mathbf{1 9 / I X / 8 5}$ & 8 & 0.24142 & 0.10014 & 0.12522 & 0.10285 \\
\hline \multicolumn{2}{|c|}{$\begin{array}{c}\text { Desviación estándar } \\
\text { ponderada }\end{array}$} & 0.24385 & 0.29237 & 0.19839 & 0.26634 \\
\hline
\end{tabular}

En la fig. 12 se observa la variación del error $\varepsilon$ con respecto al periodo $T$ de los datos sin ajustar y los corregidos usando las ecuaciones de ajuste. En general, el ajuste es adecuado; sin embargo, para el evento del 9 de octubre de 1995 se observa que la tendencia de la nube de puntos no es horizontal e igual a cero. Esto puede deberse a lo explicado anteriormente respecto a este sismo. 

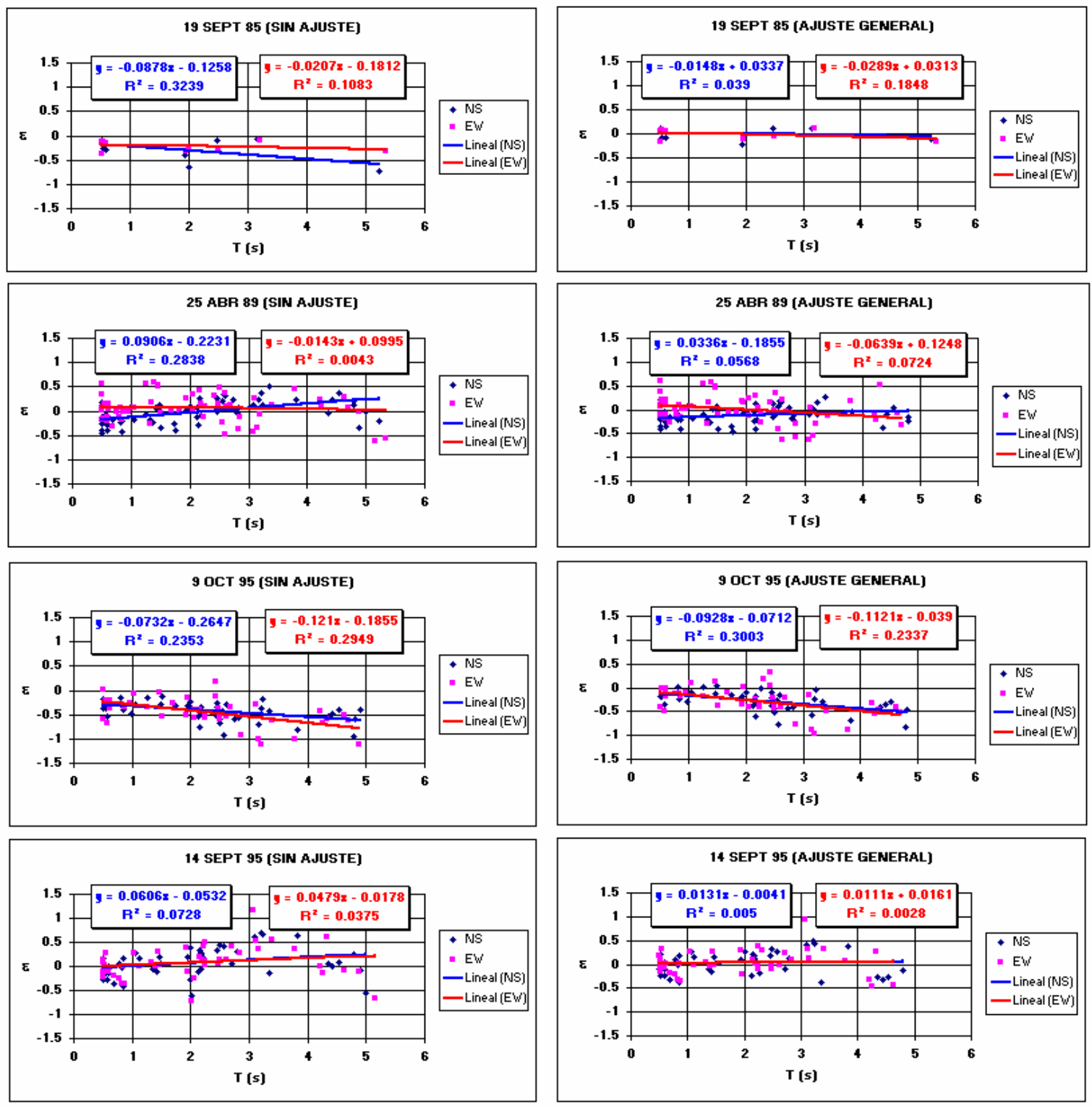

Figura 12. Distribución del error $\varepsilon$ con y sin ajuste general, para sismos postulados

En resumen, se propone que una estimación de la VMS para sismos postulados, $V_{c}$, puede obtenerse de la siguiente manera:

$V_{c}=V_{z} \cdot e^{(\alpha \cdot T+\beta)}$

donde $V_{z}$ es la VMS obtenida con el programa Z mediante aplicación directa de las ecs. 1 a 7, $T$ es el periodo del sitio y $\alpha$ y $\beta$, que dependen de la magnitud del sismo, están dados en la tabla 6. 
Con el ajuste propuesto anteriormente se pueden calcular mapas de VMS para sismos postulados. En la figs. 13 y 14 se presentan los mapas de VMS, para las componentes N-S y E$\mathrm{W}$, correspondientes a un sismo postulado con magnitud igual a 8.4 y distancia focal igual a 300 $\mathrm{km}$. Al comparar los valores de estos mapas con los obtenidos para el sismo de 1985 se aprecia un incremento en los valores de VMS debidos al aumento en el valor de la magnitud (de 8.1 a 8.4). También se aprecia que, en la mayor parte de la ciudad, la componente E-W tiene valores de VMS superiores a los de la componente N-S.

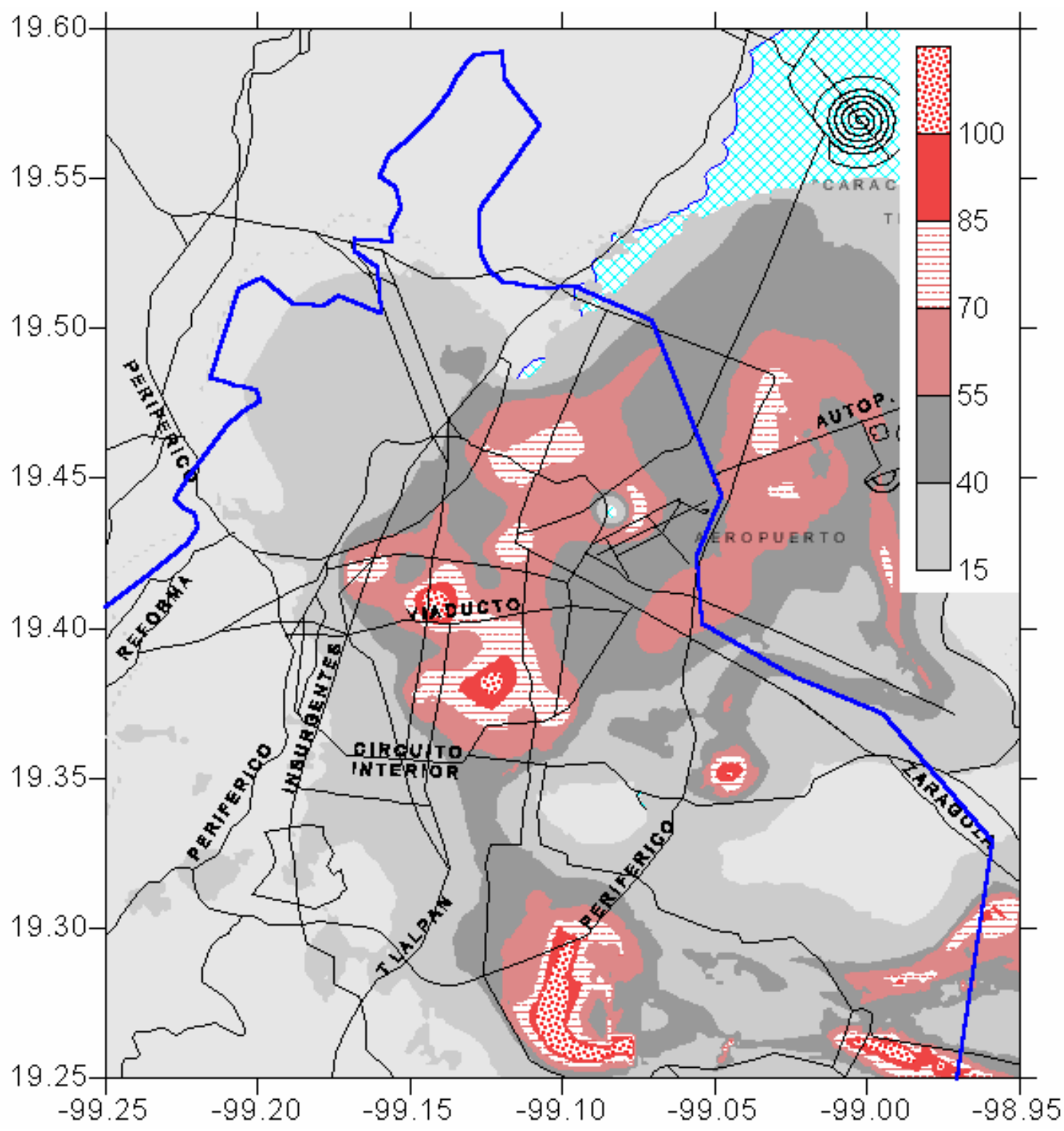

Figura 13. Mapa de VMS para un sismo postulado con m = 8.4 y R=300 km (N-S) $(\mathrm{cm} / \mathrm{s})$ 


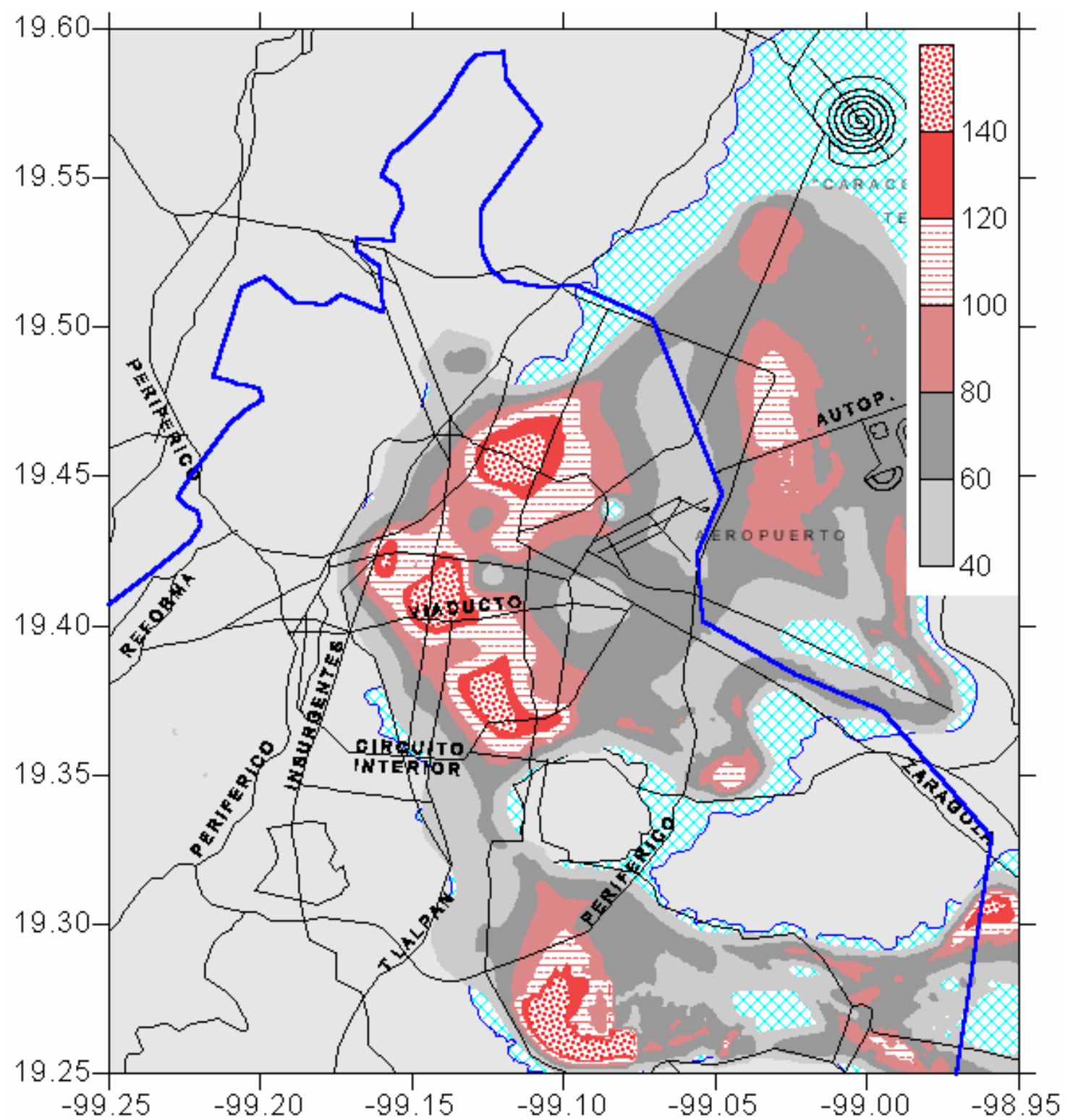

Figura 14. Mapa de VMS para un sismo postulado con m = 8.4 y R=300 km (E-W) $(\mathrm{cm} / \mathrm{s})$

\section{CONCLUSIONES}

La principal conclusión de este artículo es que los mapas de velocidad máxima del suelo presentados permiten estimar el valor esperado de este parámetro en sitios donde no hay registros sísmicos disponibles, para los sismos de interés. Esto pudo lograrse gracias a la información, relacionada con el movimiento sísmico en el valle de México, contenida en el programa $\mathrm{Z}$ y a las modificaciones y ajuste propuestos en este artículo. En estos mapas se puede visualizar la 
amplificación dinámica típica de la zona de lago, ya que en esa región los valores de VMS son significativamente superiores a los presentes en las demás zonas. Además, se pudo comprobar que los valores se incrementan con la magnitud del evento sísmico; y por otro lado, reducen su valor con la distancia focal.

Finalmente, debido a que la VMS puede ser usada para estimar la deformación del suelo, los mapas mostrados pueden servir como herramienta para el análisis de daños en líneas vitales; ya que este tipo de estructuras son afectadas por el movimiento relativo del suelo causado por la propagación de ondas sísmicas.

\section{REFERENCIAS}

Boore, D M y W B Joyner (1984), “A note on the use of random vibration theory to predict peak amplitude of transient signals”, Bulletin of the Seismological Society of America, Vol. 74, pp. 2035-2039.

Lancaster, P y K Salkauskas (1986), Curve and surface fitting. An introduction. Academy Press, Londres.

Lermo, J, R Cabrera, G Cesati y R Ortega (1990), "Estimación del periodo dominante del subsuelo en 18 sitios del Distrito Federal”, XV Reunión Nacional de Mecánica de Suelos, Innovaciones en Geotecnia, Sociedad Mexicana de Mecánica de Suelos, San Luis Potosí, México.

Newmark, N M (1967), "Problems in wave propagation in soil and rocks", Proceedings of International Symposium on Wave Propagation and Dynamic Properties of Earth Materials, Albuquerque, Nuevo México, pp. 7-26.

Oppenheim, A y R Schafer (1975), Digital signal processing, Prentice-Hall, U.S.A.

Ordaz, M (1994) “Espectros de sitio y temblores de diseño en el Distrito Federal”, Programa Z, proyecto 6534, patrocinado por el Departamento del Distrito Federal.

Ordaz, M, E Reinoso, S Singh, E Vera y J Jara (1989), "Espectros de respuesta en diversos sitios del valle ante temblores postulados en la brecha de Guerrero", VIII Congreso Nacional de Ingeniería Sísmica y VII Congreso Nacional de Ingeniería Estructural, Acapulco, México, A187A198.

Ordaz, M y S Singh (1992), "Source spectra and spectral attenuation of seismic waves from Mexican earthquakes and evidence of amplification in the hill zone of Mexico City", Bulletin of the Seismological Society of America, Vol 82, pp. 24-43.

Ordaz, M, S Singh y A Arciniega (1994), "Bayesian attenuation regressions: an application to Mexico City”, Geophys J. Int., 177, pp. 335-344.

Ordaz, M, L E Perez-Rocha, E Reinoso, C Montoya, y J Arboleda (1996), "Programa Z”, Instituto de Ingeniería, UNAM.

Pelto, C, T Elkins y H Boyd (1988), “Automatic contouring of irregularly spaced data”, Geophysics, Vol 33, pp. 424-430. 
Perez-Rocha, L E (1998), "Respuesta sísmica estructural: Efectos de sitio e interacción sueloestructura (aplicaciones al valle de México)” Tesis doctoral, DEPFI, UNAM, México.

Pineda, O y M Ordaz (2002), “Análisis de vulnerabilidad sísmica en la red primaria de distribución de agua potable del Distrito Federal”, XIII Congreso Nacional de Ingeniería Estructural, Sociedad Mexicana de Ingeniería Estructural, No. 17, pp. 719-729. Puebla, Puebla, México.

Pineda, O y M Ordaz (2003), "Seismic vulnerability function for high-diameter buried pipelines: Mexico City's primary water system case”, 2003 ASCE International Conference on Pipeline Engineering and Construction, Vol. 2, pp. 1145-1154.

Singh, S y M Ordaz (1994), “Sismicidad y movimientos fuertes en México: una visión actual”, Cuaderno de Investigación No. 18, Centro Nacional de Prevención de Desastres.

Trifunac, M D y M I Todorovska (1997), "Northridge, California, earthquake of 1994: density of red-tagged buildings versus peak horizontal velocity and intensity of shaking”, Soil Dynamics and Earthquake Engineering, Vol. 16, No. 3, pp. 209-222.

Wu, Y M, T C Shin y C H Chang (2001), "Near real-time mapping of peak ground acceleration and peak ground velocity following a strong earthquake”, Bulletin of the Seismological Society of America, Vol. 91, No. 5, pp. 1218-1228. 